\title{
Maceration of Extra Virgin Olive Oil with Common Aromatic Plants Using Ultrasound-Assisted Extraction: An UV-Vis Spectroscopic Investigation
}

\author{
Ozren Jović (D), Iva Habinovec, Nives Galić (D), and Marijan Andrašec \\ Department of Chemistry, Faculty of Science, University of Zagreb, Horvatovac 102A, 10000 Zagreb, Croatia \\ Correspondence should be addressed to Ozren Jović; ozren_jovic@yahoo.com
}

Received 26 January 2018; Revised 2 March 2018; Accepted 4 March 2018; Published 8 April 2018

Academic Editor: Jose S. Camara

Copyright (C) 2018 Ozren Jović et al. This is an open access article distributed under the Creative Commons Attribution License, which permits unrestricted use, distribution, and reproduction in any medium, provided the original work is properly cited.

\begin{abstract}
Rosemary (Rosmarinus officinalis), garden sage (Salvia officinalis), summer savory (Satureja hortensis), laurel (Laurus nobilis), and other aromatic plants were put in olive oil and exposed to ultrasounds for different duration. Filtrated oils were dissolved in cyclohexane, and UV-Vis measurements were carried out. Absorbance values corresponding to chlorophylls, carotenoids, flavonoids $(370 \mathrm{~nm}$ ), and polyphenols (around $300 \mathrm{~nm}$ ) were measured. In addition, for some samples, total phenols were determined using Folin-Denis reagent and compared with the similar maceration procedure in water solvent (instead of olive oil). Maceration without ultrasound in olive oil for each plant was also compared with ultrasound-assisted extraction. The results show that significant amount of aromatic content can be extracted in olive oil by applying ultrasounds for only few minutes, especially for Salvia officinalis powder. The use of UV-Vis measurements is simple but enough to examine the extent of phenol content extraction through such maceration procedure.
\end{abstract}

\section{Introduction}

Extra virgin olive oil is a well-known high-price health beneficial nutrient due to its fatty acid content rich of oleic acid in form of triacylglycerols, polyphenolic content, and vitamin E. It is harvested and produced mostly on the Mediterranean coast, where many other aromatic plants grow, such as rosemary, dalmatian sage, common myrtle, and laurel [1]. These aromatic plants contain essential oil rich of aroma, small volatile molecules, polyphenols, and very important antioxidant molecules. Rosemary has a bitter, astringent taste, it is used in traditional Mediterranean cuisine, and it is well known of its high antioxidant content. Garden sage is a member of the mint family Lamiaceae native to the Mediterranean region. Sage leaf contains up to 3\% essential oil, about 3.5\% hydroxycinnamic acid derivatives such as caffeic acid dimer rosmarinic acid and other tannins, phenolic diterpenes (carnosic acid), triterpenes (ursolic acid), flavonoids (ca. 1.1\%) principally flavones and their glycosides, phenolic glycosides such as coumaric acid, polysaccharides, and other substances such as benzoic acid derivatives and phytosterols [2].
It is already known that maceration of these plants can lead to enrichment of extra virgin olive oil which increases its microconstituent levels [3]. Conventional maceration could be organic solvent extraction prior to addition to food matrix or steam distillation of essential oil. Both procedures are either too long or demand too high temperature which causes a damage of the plant material. Ultrasound-assisted maceration is one of the novel procedures for the extraction of beneficial content in aromatic plants, and its use in organic solvents was already considered in the literature $[4,5]$. On the other hand, there is only one article in literature where direct aromatization of olive oil was carried out in ultrasound bath [6]. The authors considered extraction of only volatiles from basil leaves (but not, e.g., total phenol or chlorophyll content) and measured the extent of such maceration with Headspace SPME-GC-MS system. For olive oil as an extraction solvent, only the one specific plant case (of basil Ocimum basilicum) has been so far considered in literature.

Organic compounds with chromophores can be quantitatively measured by ultraviolet-visible (UV-Vis) spectroscopy. Measured region between 200 and $800 \mathrm{~nm}$ is assigned 
to electronic transitions of polyphenolic compounds, flavonoids, carotenoids, and chlorophylls. All these compounds can be extracted during maceration both in solvents and in edible oils, and most of these could be quantitatively determined [7, 8]. Chlorophylls could reduce the risk of colon cancer, especially in case of consumption of meat containing high heme concentration $[9,10]$. Carotenoids could have protective effect against lung, head, and neck cancer $[11,12]$. To the best of our knowledge, chlorophyll and carotenoid content was not evaluated using UV-Vis spectra of macerated edible oil samples. Folin-Ciocalteu reagent (or FolinDenis reagent) was used for determination of total phenols in virgin olive oils [8], but not till today in aromatized olive oil samples. The task of this study was to apply simple but extensive UV-Vis spectroscopic measurements for evaluation of extent of maceration process during different maceration time. Both conventional and ultrasound-assisted extractions of plant material directly in olive oil were performed. Determination of necessary extraction time for aromatic herbs using ultrasound is highly important for health since too long maceration could lead to degradation of edible oils, and too short time does not lead to sufficient extraction of plant substances into the oil.

In this article, we present the results of direct maceration in extra virgin olive oil of rosemary (Rosmarinus officinalis), garden sage (also known as a common sage, Salvia officinalis), laurel (Laurus nobilis), summer savory (Satureja hortensis), and other plant materials, but with accent on fast and simple UV-Vis measurements as a methodology for fast screening procedure of extent of ongoing extraction process, for maceration condition optimization purposes.

\section{Materials and Methods}

2.1. Materials. Extra virgin olive oil was obtained near Lake Vrana (Croat. Vransko Jezero) in Dalmatia, Croatia. Olives, from which olive oil was made, were grown without chemical safeners and fertilizers. Olives were ripe and were obtained during the late harvest in November 2016. Olive oil was processed within 24 hours in oil mill "Pakoška" using Pieralissi three-phase process. The oil was stored in dark glass containers at $15^{\circ} \mathrm{C}$.

Samples of rosemary powder, common fennel (Foeniculum vulgare) powder, lemon myrtle (Backhousia citriodora) powder, basil leaves (Ocimum basilicum), wild thyme (Thymus serpyllum) leaves, garden sage leaves, and summer savory leaves were bought from Harissa spice store, Armina Šrapca 8, 10000 Zagreb, Croatia, and all samples have EU origin; laurel powder and garden sage powder were bought from Farma shop, Medulićeva 18, Zagreb, EU origin; nettle leaves (Urtica dioica) and lemon grass (Cymbopogon citratus) were obtained from Suban d.o.o., 10434 Strmec, Croatia.

2.2. Content of Olive Oil. The content of olive oil sample was determined using GC-MS, HPLC, FTIR, NMR, and UV-Vis spectroscopy, and wet analytical methods were also carried out in our laboratory at the Faculty of Science. It should be noted that measurements were conducted four months after obtaining olive oil in oil mill. Fatty acid content was determined using GC-MS with the help of NMR spectroscopy. Iodine value was calculated using FTIR and NMR spectroscopy [13]. Diacylglycerols were calculated with the use of NMR spectroscopy [14]. Free fatty acid content (ISO 660:2009(E)), peroxide value (AOAC official method 965.33), and induction period using standard Rancimat method at $120^{\circ} \mathrm{C}$ were determined too, along with absorbance in UV (CODEX STAN 33-1981).

2.3. Effect of Ultrasound on Olive Oil. Two samples of $5 \mathrm{~g}$ of olive oil were put in separate $15 \mathrm{~mL}$ glass bottle, and one of them was put into the ultrasonic bath at $49-51^{\circ} \mathrm{C}$. Ultrasounds were applied at intensity of $<1 \mathrm{~W} / \mathrm{cm}^{2}$ and frequency of $40 \mathrm{kHz}$. The other sample was reference sample. Both samples were FT-IR sampled on ATR crystal in the region $4000-600 \mathrm{~cm}^{-1}$ with $2 \mathrm{~cm}^{-1}$ nominal resolution. Their peroxide values and free fatty acids were determined, along with $\mathrm{K} 270$ and $\Delta K$ values.

2.4. Conventional Maceration. Approximately $0.75 \mathrm{~g}$ of dry plant sample was put in $5 \mathrm{~g}$ of olive oil in $15 \mathrm{~mL}$ glass bottles.After a considerable amount of time (at least one hour and at most two weeks), the macerate was filtrated and stored at $23-25^{\circ} \mathrm{C}$ for further analysis. The result of maceration for the particular plant during different maceration time was determined by separate experiments. For example, if for the same plant material there were 5 different maceration times, then five separate experiments have been carried out.

2.5. Ultrasound Maceration. Approximately $0.75 \mathrm{~g}$ of dry plant sample was put in $5 \mathrm{~g}$ of olive oil in $15 \mathrm{~mL}$ glass bottle. The bottle was immediately put in ultrasonic bath Bandelin Sonorex, and the ultrasounds were applied at intensity of ultrasounds $<1 \mathrm{~W} / \mathrm{cm}^{2}$ and frequency of $40 \mathrm{kHz}$. The temperature was $23-25^{\circ} \mathrm{C}$. After a considerable amount of time (at least one minute and at most one hour), the macerate was filtrated and stored at $23-25^{\circ} \mathrm{C}$ for further analysis. Each experiment was conducted separately (i.e., if for the same plant material there were 5 different maceration times, then five separate experiments have been carried out).

For comparison purpose, approximately $0.75 \mathrm{~g}$ of dry plant sample was put in $5 \mathrm{~mL}$ of redistilled water in $15 \mathrm{~mL}$ glass bottle. The bottle was immediately put in ultrasonic bath Bandelin Sonorex, and the ultrasounds were applied at intensity of ultrasounds $<1 \mathrm{~W} / \mathrm{cm}^{2}$ and frequency of $40 \mathrm{kHz}$. The temperature was $23-25^{\circ} \mathrm{C}$. After 15 minutes, macerate was filtrated and stored at $23-25^{\circ} \mathrm{C}$ for further analysis.

2.6. UV-Vis Measurements. UV-Vis measurements were carried out on Cary 5 instrument. The spectra were recorded in the range between 200 and $800 \mathrm{~nm}$ using $10 \mathrm{~mm}$ path cells in the UV acquisition compartment (temperature $25.00 \pm$ $0.02^{\circ} \mathrm{C}$ ) with resolution of $1 \mathrm{~nm}$ yielding 601 spectral data points. UV-Vis spectrum of cyclohexane (Merck) was recorded as baseline and subtracted from each acquired spectrum. Approximately $1 \mathrm{~g}$ of oil macerate sample was dissolved in $10 \mathrm{~mL}$ cyclohexane. UV-Vis measurements were performed twice for each sample. 
After the measurements, chlorophyll content was calculated as follows [15]:

total chlorophylls $=34.53 \times F \times(\mathrm{A} 670-0.5 \times(\mathrm{A} 630+\mathrm{A} 710))$,

where $F$ equals the division factor between pure oil sample and $1 \mathrm{~g}$ of olive oil sample dissolved in $10 \mathrm{~mL}$ of cyclohexane; in our study, it equals 9.62. Content is expressed as content of chlorophyll pigments in $\mathrm{mg}$ of pheophytin in a $1 \mathrm{~kg}$ of oil.

According to references $[7,15]$, carotenoids were determined according to the following equation:

$$
\begin{aligned}
\text { total carotenoids }= & 34.53 \times 9.62 \times \frac{613}{2000} \\
& \times(\mathrm{A} 467-0.5 \times(\mathrm{A} 630+\mathrm{A} 710)) .
\end{aligned}
$$

2.7. Determination of Total Phenols. The selected oil macerate samples were dissolved in hexane $(1 \mathrm{~g}$ of oil in $5 \mathrm{~mL}$ solvent) and extracted three times with $3 \mathrm{~mL}$ aqueous methanol $\left(\mathrm{V}\left(\mathrm{CH}_{3} \mathrm{OH}\right): \mathrm{V}\left(\mathrm{H}_{2} \mathrm{O}\right)=80: 20\right)$, and finally, the extract was made up to $10 \mathrm{~mL}$ with redistilled water and left to stand overnight. Water macerate samples were used as they are (or somewhat diluted in water). The $1 \mathrm{~mL}$ of extract (or smaller volume (especially for water macerates), depending on the final UV-Vis spectrum) was pipetted to eprouvette, and $5 \mathrm{~mL}$ of redistilled water was added. Then, $0.5 \mathrm{~mL}$ of FolinDenis reagent was added and the eprouvette was shaken. Few minutes later, $1 \mathrm{~mL}$ of $20 \% \mathrm{Na}_{2} \mathrm{CO}_{3}$ was added and the eprouvette was well shaken. After at least 1 hour, the eprouvette was centrifuged and the UV-Vis spectrum of sample was recorded in the region $600-800 \mathrm{~nm}$ with $1 \mathrm{~nm}$ resolution against the blank sample of redistilled water. To $1 \mathrm{~mL}$ of standard solutions of gallic acid at concentrations of 0 (redistilled $\mathrm{H}_{2} \mathrm{O}$ sample), $10,20,30,40$, and $50 \mathrm{mg} / \mathrm{kg}$, the following chemicals were added: $5 \mathrm{~mL}$ water, $0.5 \mathrm{~mL}$ Folin-Denis reagent, and $1 \mathrm{ml} \mathrm{Na} \mathrm{CO}_{3}$. Each standard solution was centrifuged prior to UV-Vis measurement. The absorbance value was read at $760 \mathrm{~nm}$, and the exact amounts of polyphenols in pure olive oil and in considered macerate samples were determined using calibration curve.

2.8. HPLC Determination of Alpha-Tocopherol (Vitamin E). Approximately $1 \mathrm{~g}$ of oil was extracted three times with $3 \mathrm{~mL}$ pure of methanol, and methanol was added to a final volume of $10 \mathrm{~mL}$. Methanol from methanolic extract was evaporated in rotatory evaporator under reduced pressure, and $1 \mathrm{~mL}$ of methanol was added to obtain more concentrated solution. It was filtrated through $0.45 \mu \mathrm{m}$ PTFE filter into the $2 \mathrm{~mL}$ HPLC vial. HPLC measurement was carried out with Agilent 1100 with quaternary pump, thermostated autosampler, thermostated column compartment, and DAD detector. Chromatographic separation was achieved on Agilent Eclipse XDB C18 chromatographic column $(150 \mathrm{~mm} \times 4.6 \mathrm{~mm}$; pore size $5 \mu \mathrm{m})$ using the methanol : water $=95: 5 \mathrm{v} / \mathrm{v}$ in isocratic elution mode. Flow rate was $1 \mathrm{~mL} / \mathrm{min}$. Column temperature was $35^{\circ} \mathrm{C}$, and wavelength detection was at $292 \mathrm{~nm}$.

\section{Results and Discussion}

3.1. Content of Olive Oil. The determined content of oil sample was as follows: unsaturated fatty acids $12.8 \%$, of which, 9.2\% palmitic acid and $2.6 \%$ stearic acid; monounsaturated fatty acids $74.8 \%$; polyunsaturated fatty acids $12.4 \%$, of which, omega-3 fatty acids $0.5 \%$ and omega- 6 fatty acids $11.9 \%$ (st. dev. for fatty acids $0.2 \%$ ); iodine value $86.0 \pm 0.9 ; 1,2$-diacilglycerols $2.6( \pm 0.3) \%$; 1,3 -diacilglycerols $0.4( \pm 0.1) \%$; free fatty acid expressed as mass fraction of oleic acid $0.57( \pm 0.03) \%$; peroxide value $16.8( \pm 0.5) \mathrm{meq}$ active $\mathrm{O}_{2}$; induction time $3.9( \pm 0.3)$ hours at $120^{\circ} \mathrm{C}$ for $20 \mathrm{~L} /$ $\mathrm{h}$ air stream; and absorbance in UV K270 $=0.198( \pm 0.007)$, $\mathrm{K} 232=2.183( \pm 0.012)$, and $\Delta K=0.0026( \pm 0.0004)$; total chlorophylls $8.7( \pm 0.7) \mathrm{mg} / \mathrm{kg}$ expressed as pheophytin; and total carotenoids $9.67( \pm 0.82) \mathrm{mg} / \mathrm{kg}$.

3.2. Effect of Ultrasound to Olive Oil. A comparison of sample exposed $3 \mathrm{~h}$ at $50^{\circ} \mathrm{C}$ to ultrasound with a referent sample resulted in no difference between free fatty acid content and negligible difference in peroxide value (referent sample even had slightly higher PV). Ratio of ATR intensity at $966 \mathrm{~cm}^{-1}$ and $722 \mathrm{~cm}^{-1}\left(I\left(967 \mathrm{~cm}^{-1}\right) / I\left(722 \mathrm{~cm}^{-1}\right)\right)$ was even slightly higher for referent sample, which means that ultrasound does not lead to formation of transdouble bonds. Both samples had $\mathrm{K} 270<0.22( \pm 0.01)$ (although sample exposed to ultrasound had $\approx 0.01$ higher value) and $\Delta K<0.01$ (referent sample had negligibly higher value). These results clearly show that the ultrasound at $<1 \mathrm{~W} / \mathrm{cm}^{2}$ does not alter quality or content of extra virgin olive oil, at least not from analytical point of view (organoleptic analysis was not performed).

3.3. Plant Maceration: General Results. The absorbance values of olive oil samples after conventional and ultrasound maceration at different wavelengths are shown in Table 1. As can be seen, the ultrasound-assisted maceration had clear advantage over conventional one since in most cases, higher absorbance values were obtained.

Only 10 to 15 minutes of ultrasound was sufficient for the extraction of necessary material from most of studied plants to olive oils. From Table 1, it can be also seen that the effect of ultrasound was more accentuated for powder samples than for leaves; a clear example is garden sage leaves (5) and garden sage powder (8) (Table 1). Additional important notice is that for the same extraction time period, extent of maceration depends on sample particle size [16]; we therefore present picture of each sample in Supplementary Materials (available here).

3.4. Plant Maceration: Specific Results. Figure 1 displays the UV-Vis spectra of olive oil after conventional maceration of rosemary powder at different extraction time, increase in total chlorophylls and carotenoids content, and changes of absorbance at selected wavelengths. Figure 2 shows analogue results for ultrasound-assisted maceration. As can be seen, two weeks of constant maceration can attain lower levels of chlorophylls and carotenoids along with A415, A370, and A300 values. A415 represents the peak maximum in Vis region and is assigned mostly to carotenoids, A370 approximately describes flavonoid content [17], and A300 could 
TABLE 1: The absorbance of olive oil samples after maceration at selected wavelengths (relative standard deviation $4 \%$ ).

\begin{tabular}{|c|c|c|c|c|c|c|c|}
\hline & Maceration & Duration & A670 & A470 & A415 & A370 & A300 \\
\hline \multicolumn{3}{|c|}{ Pure (unmacerated) olive oil sample } & 0.0259 & 0.0870 & 0.1248 & 0.0884 & 0.5749 \\
\hline \multirow{2}{*}{ (1) Rosemary powder } & Conventional & $20 \mathrm{~h}$ & 0.0839 & 0.1347 & 0.2985 & 0.2129 & 1.3177 \\
\hline & Ultrasound & $10 \mathrm{~min}$ & 0.1915 & 0.2476 & 0.6415 & 0.4672 & 2.2426 \\
\hline \multirow{2}{*}{ (2) Common fennel powder } & Conventional & $20 \mathrm{~h}$ & 0.0626 & 0.1061 & 0.2317 & 0.1613 & 2.6744 \\
\hline & Ultrasound & $15 \mathrm{~min}$ & 0.0698 & 0.1180 & 0.2650 & 0.1943 & 2.2370 \\
\hline \multirow{2}{*}{ (3) Lemon myrtle powder } & Conventional & $20 \mathrm{~h}$ & 0.0764 & 0.0994 & 0.2506 & 0.1646 & 0.8484 \\
\hline & Ultrasound & $15 \mathrm{~min}$ & 0.0840 & 0.1129 & 0.2871 & 0.1996 & 0.8581 \\
\hline \multirow{2}{*}{ (4) Wild thyme leaves } & Conventional & $20 \mathrm{~h}$ & 0.1317 & 0.1826 & 0.3986 & 0.2345 & 0.8838 \\
\hline & Ultrasound & $15 \mathrm{~min}$ & 0.0741 & 0.1404 & 0.2671 & 0.1950 & 0.8814 \\
\hline \multirow{2}{*}{ (5) Garden sage leaves } & Conventional & $20 \mathrm{~h}$ & 0.0571 & 0.1047 & 0.2172 & 0.1832 & 1.2365 \\
\hline & Ultrasound & $15 \mathrm{~min}$ & 0.0384 & 0.0975 & 0.1694 & 0.1411 & 0.9427 \\
\hline \multirow{2}{*}{ (6) Summer savory leaves } & Conventional & $8 \mathrm{~h}$ & 0.0815 & 0.1228 & 0.2620 & 0.1644 & 0.6307 \\
\hline & Ultrasound & $10 \mathrm{~min}$ & 0.1061 & 0.1552 & 0.3429 & 0.2210 & 0.6436 \\
\hline \multirow{2}{*}{ (7) Laurel powder } & Conventional & $20 \mathrm{~h}$ & 0.2163 & 0.1624 & 0.5881 & 0.3348 & 0.8581 \\
\hline & Ultrasound & $15 \mathrm{~min}$ & 0.9808 & 0.4258 & 2.4472 & 1.3271 & 1.9213 \\
\hline \multirow{2}{*}{ (8) Garden sage powder } & Conventional & $20 \mathrm{~h}$ & 0.0611 & 0.1152 & 0.2642 & 0.2320 & 1.1561 \\
\hline & Ultrasound & $10 \mathrm{~min}$ & 0.1524 & 0.1688 & 0.5267 & 0.5075 & 2.0867 \\
\hline \multirow{2}{*}{ (9) Nettle leaves } & Conventional & $72 \mathrm{~h}$ & 0.0634 & 0.1329 & 0.2368 & 0.1456 & 0.6346 \\
\hline & Ultrasound & $60 \mathrm{~min}$ & 0.0648 & 0.1566 & 0.2535 & 0.1658 & 0.6654 \\
\hline \multirow{2}{*}{ (10) Lemon grass powder } & Conventional & 7 days & 0.5572 & 0.1406 & 1.3329 & 0.8488 & 1.3519 \\
\hline & Ultrasound & $60 \mathrm{~min}$ & 0.5629 & 0.1090 & 1.2527 & 0.7785 & 1.2413 \\
\hline
\end{tabular}

represent polyphenol content since the absorbance at $280 \mathrm{~nm}$ is highly affected by K272-conjugated triene value found in olive oils [8]. Also, these absorbance values seem to be pretty well mutually correlated in case of rosemary for both conventional and ultrasound maceration. This means that only by monitoring chlorophyll values, one could also roughly predict carotenoids, flavonoids, and polyphenols. From Figure 2, one could conclude that approximately 7 minutes of ultrasound maceration is enough for the extraction of organic compounds from rosemary powder into the olive oil. Folin-Denis experiment for determination of total phenols has shown that 5 minutes of ultrasound maceration increased total phenol content by $875( \pm 30) \mathrm{mg} / \mathrm{kg}$ and 10 minutes of ultrasound maceration by $933( \pm 30) \mathrm{mg} / \mathrm{kg}$ (i.e., total phenol content was then $1023( \pm 30) \mathrm{mg} / \mathrm{kg}$ and $1081( \pm 30) \mathrm{mg} / \mathrm{kg}$ for 5 and 10 minutes of ultrasound, resp.). A very high absorbance (Table 1) recorded for samples after 10 minutes of ultrasound maceration of rosemary powder $(\mathrm{A} 300=2.24)$ was accompanied with determined increase in total phenol content. However, in water (instead of olive oil) as an extraction solvent, the total phenol for rosemary content obtained was $2905( \pm 80) \mathrm{mg} / \mathrm{kg}$. This means that the extraction in water is almost by factor of 3 more effective than that in oil, but on the other hand, in water, no peak chlorophyll or carotenoid peaks were observed.

Figure 3 describes results for ultrasound-assisted maceration of laurel powder in olive oil. Only one minute of ultrasound maceration was sufficient to achieve higher absorbance values than after 20 hours of conventional maceration. In the case of laurel maceration, chlorophyll, and carotenoid content, A415, A370, and A300 were also mutually highly correlated. Some discrepancy of results obtained after 2 and 3 minutes of ultrasound application could be a consequence of powder inhomogeneity and different interaction of powder with the oil in these two maceration experiments. After 5 minutes of ultrasound-assisted maceration, there was no significant difference in UV-Vis spectra. So, it can be concluded, from this time frame, that the maximum extraction was achieved only in 5 minutes of ultrasound experiment! Folin-Denis experiment has shown that total phenol content in that sample amounted to $396( \pm 20) \mathrm{mg} / \mathrm{kg}$, which was significantly higher than that in unmacerated oil. The total content of phenols in pure olive oil was $148( \pm 10) \mathrm{mg} / \mathrm{kg}$. An increase in A300 of 1.3 (Figure 3) was observed along with the increase of total phenol content. In water (instead of olive oil) as an extraction solvent, the total phenol for laurel obtained was $2256( \pm 70) \mathrm{mg} / \mathrm{kg}$. This means that extraction in water is almost by factor of 5.5 more effective than that in oil, but with the absence of chlorophyll or carotenoid peaks in water.

The results of conventional and ultrasound maceration of summer savory leaves are shown in Figures S1 and S2 (Supplementary Material). These results show that 15 minutes of ultrasound maceration attains approximately similar results as 24 hours of conventional maceration.

The results for ultrasound-assisted maceration for garden sage powder are presented in Figure 4. It can be seen that when compared with (Figure 4 caption), only one minute of ultrasound maceration was enough to attain higher 

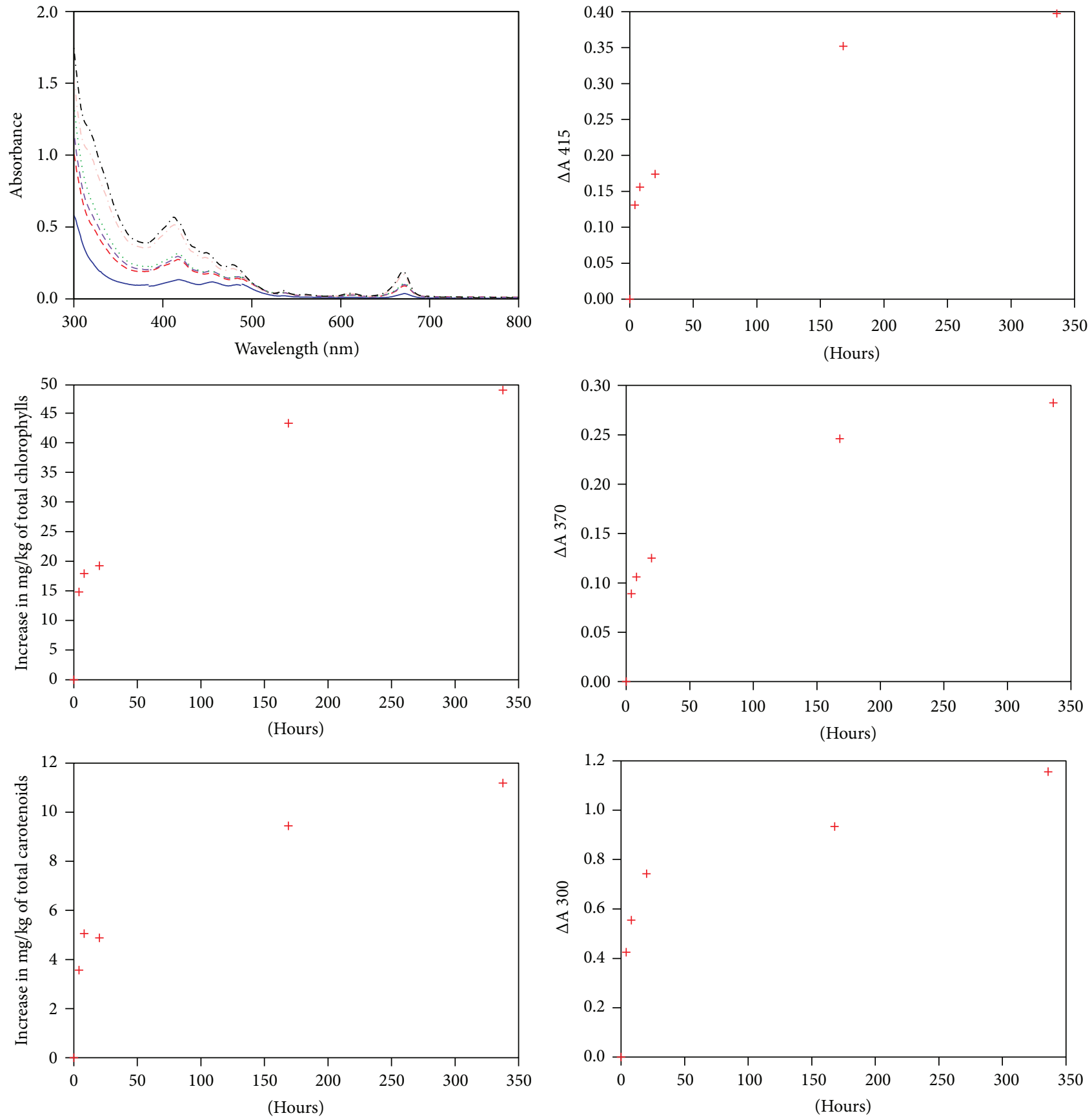

FIgURE 1: Rosemary conventional maceration, results of UV-Vis measurements.

absorbance values and higher values of chlorophylls and carotenoids in comparison to conventional maceration. After only three minutes, no significant increase in spectral variables was observed. Folin-Denis experiment has shown that total phenol content in oil sample after 1, 2, and 3 minutes of ultrasound maceration equals $812( \pm 40), 1069( \pm 40)$, and $1142( \pm 40) \mathrm{mg} / \mathrm{kg}$, respectively. A very high absorbance (Table 1) recorded for a sample after 10 minutes of ultrasound maceration of sage $(\mathrm{A} 300=2.08)$ was accompanied with an increase in total phenol content. There was no further increase in total phenolic content after 3 minutes of maceration, which is an important finding since there is no benefit with longer exposure to ultrasound. In addition, based on the simple UV-Vis measurement, the prediction of approximate increase in phenolic content can be made, as it was for rosemary samples.

In samples of pure olive oil and in oil after maceration with garden sage powder, the HPLC method was used for determination of alpha-tocopherol, one form of vitamin $\mathrm{E}$. The pure olive oil contained $46.4( \pm 5)$ ppm while the macerated one $47.8( \pm 5)$ ppm of vitamin $\mathrm{E}$, which was an insignificant increase.

Finally, in water (instead of olive oil) as an extraction solvent, the total phenol for sage content obtained was 

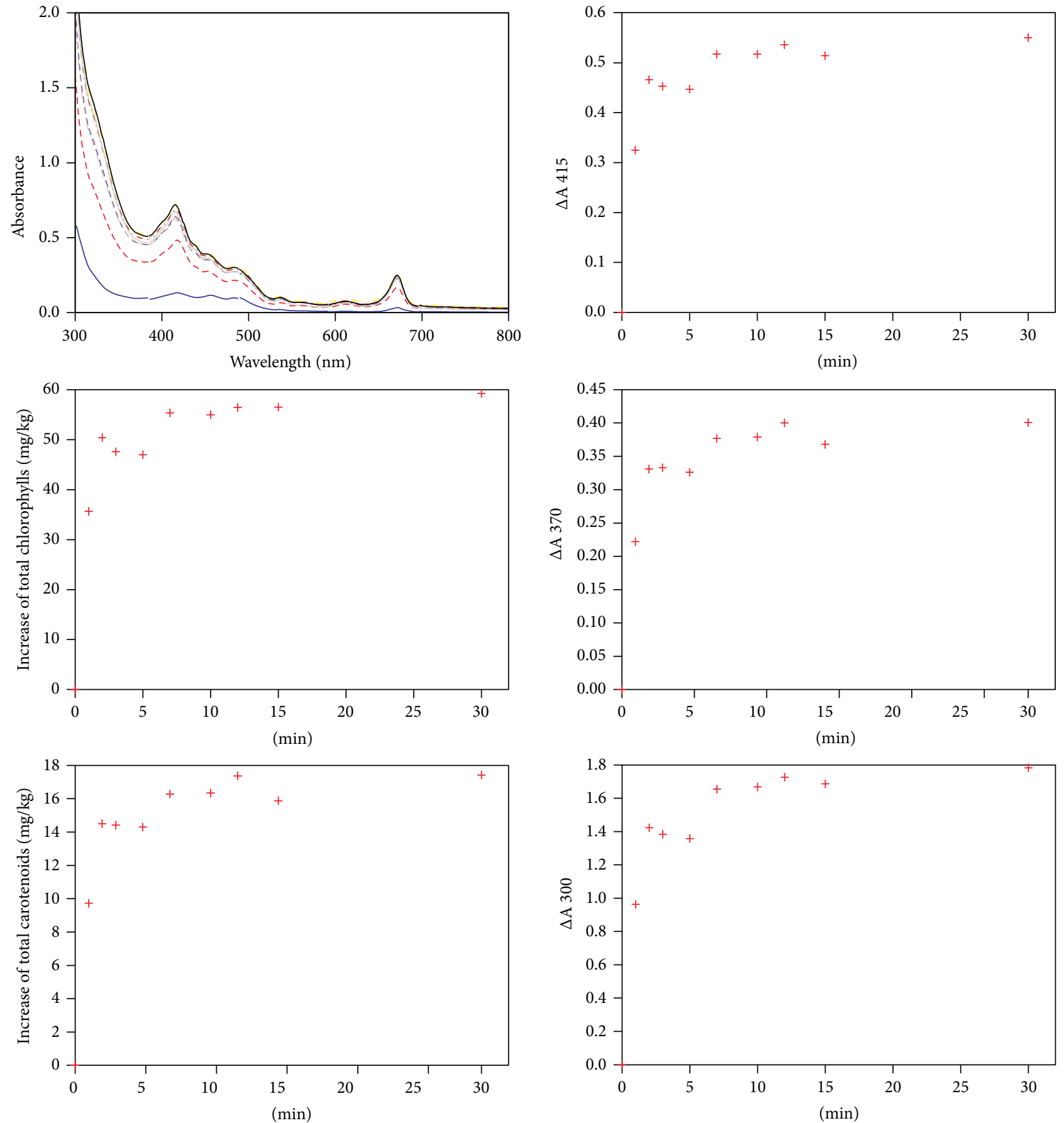

FIgURE 2: Rosemary ultrasound maceration, results of UV-Vis measurements. For comparison with conventional maceration, see Figure 1.

$1106( \pm 60) \mathrm{mg} / \mathrm{kg}$. This means that regarding phenol content, the extraction in water does not significantly differ than the extraction in olive oil, while taking into account that no chlorophyll or carotenoid peaks were observed in water.

How important this result is can be seen for other cases, since comparison in total phenol content between olive oil and water as solvents was also conducted for the following cases: lemon grass, summer savory, common fennel, lemon myrtle, and basil (Ocimum basilicum). In water as the solvent content of total extracted phenol content was the following: lemon grass $1322( \pm 60) \mathrm{mg} / \mathrm{kg}$, summer savory $5627( \pm 120)$ $\mathrm{mg} / \mathrm{kg}$, common fennel $1344( \pm 40) \mathrm{mg} / \mathrm{kg}$, lemon myrtle 4982 $( \pm 110) \mathrm{mg} / \mathrm{kg}$, and basil $1489( \pm 50) \mathrm{mg} / \mathrm{kg}$. On the other hand, in oil as a solvent for all five mentioned cases, total phenol content did not significantly differ from phenol content of olive oil (as a solvent)! This means that no significant extraction of phenols occurred at all for each of these mentioned plants in olive oil. In 6, the authors extracted volatiles from basil in olive oil and detected them with GC-MS, but these compounds are not phenols and do not react with 

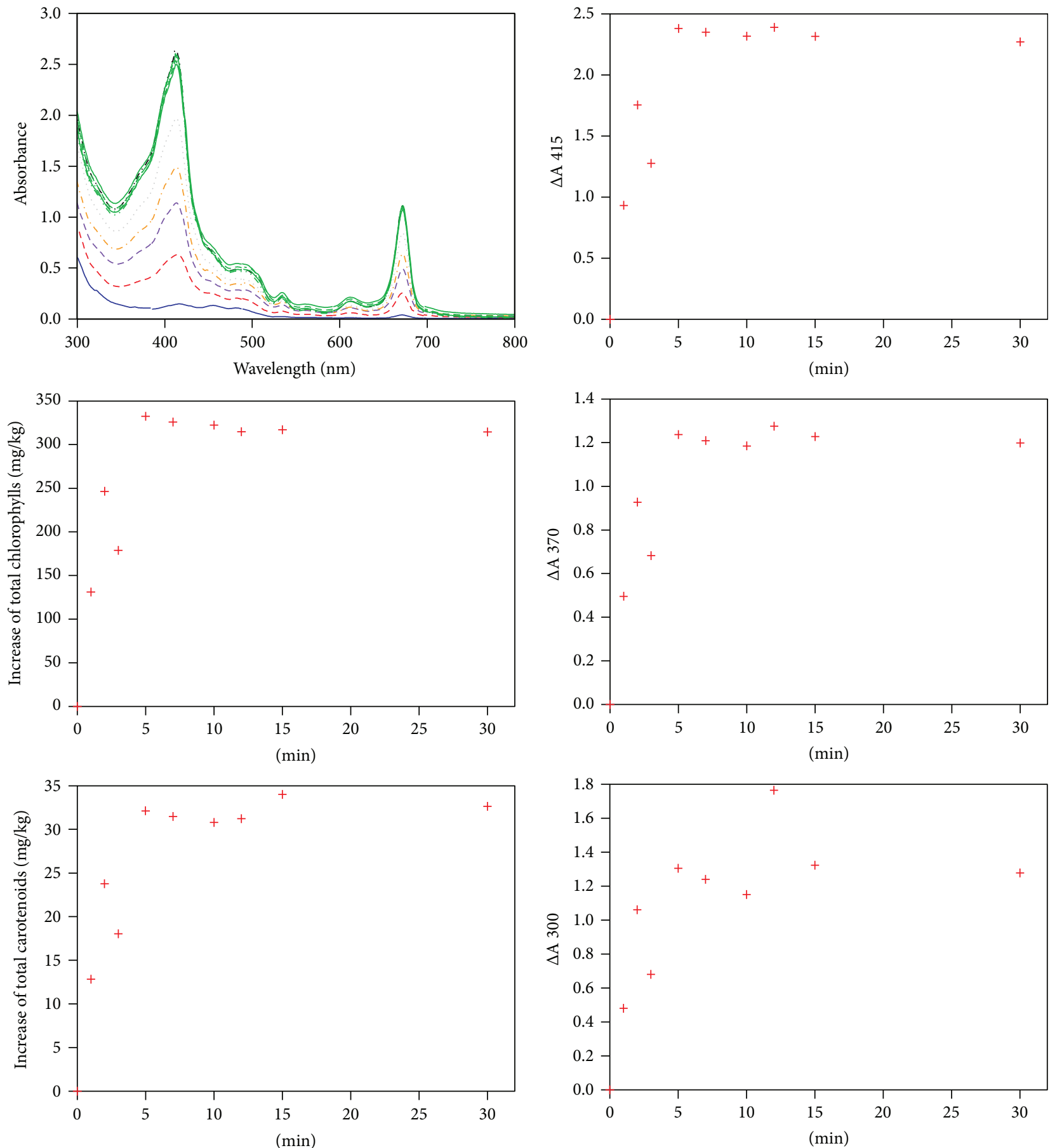

FIGURE 3: Laurel ultrasound maceration, results of UV-Vis measurements. For comparison, the results for 20 hours of conventional maceration are as follows: increase in chlorophylls, $63 \mathrm{mg}$; increase in carotenoids, $7.2 \mathrm{mg} ; \Delta A 415,0.456 ; \Delta A 370,0.236$; and $\Delta A 300,0.261$.

phenol reagents. Observed absorbance values for these plants at or below $300 \mathrm{~nm}$ (Table 1) therefore represent volatile compounds or some unsaturated components.

\section{Conclusion}

This study has shown novel results regarding ultrasound extraction of aromatic plants directly into the olive oil. For the most of studied plants, the application of ultrasound reduces the time of the whole extraction process from 24 hours or several weeks to only few minutes. In addition, even several hours of exposure of oil to ultrasound did not alter the oxidative stability of studied oil. Within several minutes of ultrasound maceration of studied garden sage, laurel, and rosemary, the high-quality products with high polyphenolic content were obtained, together with carotenoid and chlorophyll. From the UV-Vis spectrum of final product in cyclohexane solution, one can roughly predict an increase in 

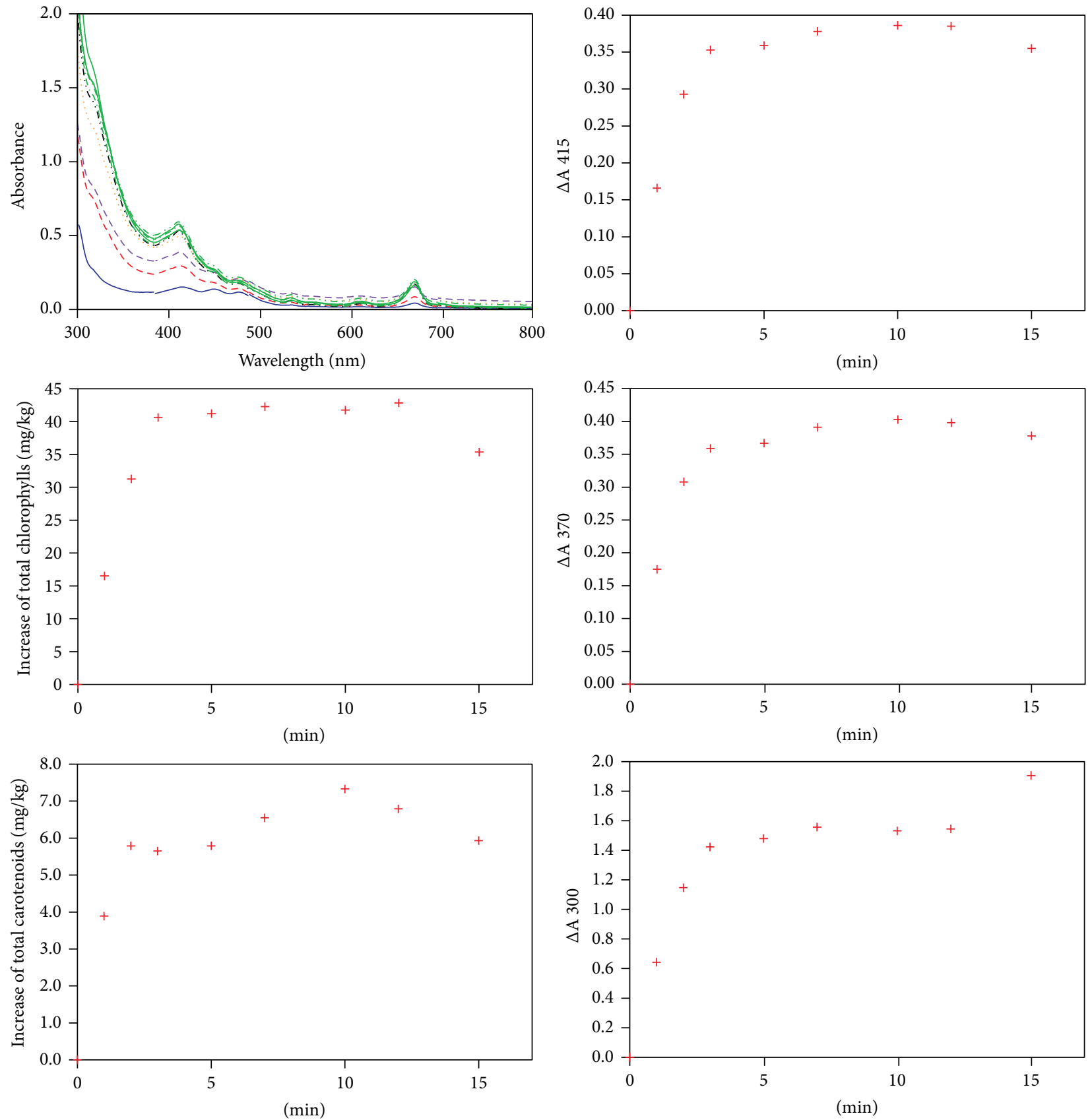

FIGURE 4: Garden sage ultrasound maceration, results of UV-Vis measurements. For comparison, the results for 20 hours of conventional maceration are as follows: increase in chlorophylls, $11.4 \mathrm{mg}$; increase in carotenoids, $1.9 \mathrm{mg} ; \Delta A 415,0.124 ; \Delta A 370,0.128 ;$ and $\Delta A 300,0.601$.

polyphenolic compounds of the measured macerate sample. An important finding is that the difference in extraction of total phenol content between water and oil as solvents is not too high for rosemary powder and even the same for garden sage powder, which is definitely not the case for the most of all other studied plant materials.

\section{Disclosure}

This article does not contain any studies with human or animal subjects.

\section{Conflicts of Interest}

Ozren Jović declares that he has no conflict of interest with the Ministry of Science who supported this research under the mentioned research project.

\section{Acknowledgments}

This work was supported by the Ministry of Science, Croatia under research project spectroscopic analysis of unsaturated systems and metal compounds MSES 119-1191342-2959. 


\section{Supplementary Materials}

The supporting information are enclosed to this version of the manuscript. Picture S1: basil leaves, Ocimum basilicum, view of used sample (scale is in centimeters). Picture S2: summer savory leaves, Satureja hortensis, view of used sample (scale is in centimeters). Picture S3: garden sage leaves, Salvia officinalis, view of used sample (scale is in centimeters). Picture S4: garden sage powder, Salvia officinalis, view of used sample (scale is in centimeters). Picture S5: common fennel powder, Foeniculum vulgare, view of used sample (scale is in centimeters). Picture S6: nettle leaves, Urtica dioica, view of used sample (scale is in centimeters). Picture S7: lemon myrtle powder, Backhousia citriodora, view of used sample (scale is in centimeters). Picture S8: lemon grass powder, Cymbopogon citratus, view of used sample (scale is in centimeters). Picture S9: laurel powder, Laurus nobilis, view of used sample (scale is in centimeters). Picture S10: wild thyme leaves, Thymus serpyllum, view of used sample (scale is in centimeters). Picture S11: rosemary powder, Rosmarinus officinalis, view of used sample (scale is in centimeters). Figure S1: summer savory conventional maceration, results of UV-Vis measurements. Figure S2: summer savory ultrasound maceration, results of UV-Vis measurements. For comparison with conventional maceration, see Figure 1 . (Supplementary Materials)

\section{References}

[1] A. C. Zeven and J. M. J. de Wet, Dictionary of Cultivated Plants and their Regions of Diversity, Centre for Agricultural Publishing and Documentation, Wageningen, 1982.

[2] P. Bradley, "Sage Leaf," in British Herbal Compedium, vol. 2, pp. 339-344, British Herbal Medicine Association, 2006.

[3] S. F. Hamed, "Edible oil as an alternate solvent for extraction of antioxidant components from natural herbs," Journal of Applied Sciences Research, vol. 2, no. 9, pp. 567-571, 2006.

[4] A. E. Ince, S. Sahin, and G. Sumnu, "Comparison of microwave and ultrasound-assisted extraction techniques for leaching of phenolic compounds from nettle," Journal of Food Science and Technology, vol. 51, no. 10, pp. 2776-2782, 2014.

[5] M. Vinatoru, "An overview of the ultrasonically assisted extraction of bioactive principles from herbs," Ultrasonics Sonochemistry, vol. 8, no. 3, pp. 303-313, 2001.

[6] S. Veillet, V. Tomao, and F. Chemat, "Ultrasound assisted maceration: An original procedure for direct aromatisation of olive oil with basil," Food Chemistry, vol. 123, no. 3, pp. 905$911,2010$.

[7] J. Cayuela, K. Yousfi, M. C. Martinez, and J. M. Garcia, "Rapid determination of olive oil chlorophylls and carotenoids by using visible spectroscopy," Journal of the American Oil Chemists' Society, vol. 91, no. 10, pp. 1677-1684, 2014.

[8] E. Fuentes, M. E. Baez, M. Bravo, C. Cid, and F. Labra, "Determination of total phenolic content in olive oil samples by UV-visible spectrometry and multivariate calibration," Food Analytical Methods, vol. 5, no. 6, pp. 1311-1319, 2012.

[9] T.E. Abbink, J. de Vogel, J. F. Bol, and H. J. Linthorst, "Induction of a hypersensitive response by chimeric helicase sequences of tobamoviruses U1 and Ob in N-Carrying tobacco," Molecular Plant-Microbe Interactions, vol. 14, no. 9, pp. 1086-1095, 2001.
[10] J. de Vogel, D. S. Jonker-Termont, E. M. van Lieshout, M. B. Katan, and R. van der Meer, "Green vegetables, red meat and colon cancer: chlorophyll prevents the cytotoxic and hyperproliferative effects of haem in rat colon," Carcinogenesis, vol. 26, no. 2, pp. 387-393, 2005.

[11] A. R. Vieira, L. Abar, S. Vingeliene et al., "Fruits, vegetables and lung cancer risk: a systematic review and meta-analysis," Annals of Oncology, vol. 27, no. 1, pp. 81-96, 2016.

[12] E. Leoncini, D. Nedovic, N. Panic, R. Pastorino, V. Edefonti, and S. Boccia, "Carotenoid intake from natural sources and head and neck cancer: a systematic review and meta-analysis of epidemiological studies," Cancer Epidemiology, Biomarkers \& Prevention, vol. 24, no. 7, pp. 1003-1011, 2015.

[13] Y. Miyake, K. Yokomizo, and N. Matsuzaki, "Rapid determination of iodine value by ${ }^{1} \mathrm{H}$ nuclear magnetic resonance spectroscopy," Journal of the American Oil Chemists' Society, vol. 75, no. 1, pp. 15-19, 1998.

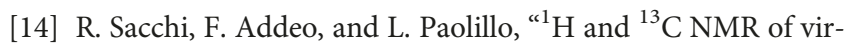
gin olive oil. An overview," Magnetic Resonance in Chemistry, vol. 35, no. 13, pp. S133-S145, 1997.

[15] J. Pokorny, L. Kalinova, and P. Dysseler, "Determination of chlorophyll pigments in crude vegetable oils: results of a collaborative study and the standardized method (technical report)," Pure and Applied Chemistry, vol. 67, no. 10, pp. 1781-1787, 1995.

[16] T. M. Takeuchi, C. G. Pereira, M. E. M. Braga, M. R. Maróstica Jr., P. F. Leal, and M. Angela, "Low-pressure solvent extraction (solid-liquid extraction, microwave assisted, and ultrasound assisted) from condimentary plants," in Extracting Bioactive Compounds for Food Products: Theory and Applications (Contemporary Food Engineering), M. Angela and A. Meireles, Eds., CRC Press, Boca Raton, FL, USA, 1st edition, 2008.

[17] April 2017, http://www.shimadzu.com/an/hplc/support/lc/ap/ n9j25k00000bfu20.html. 

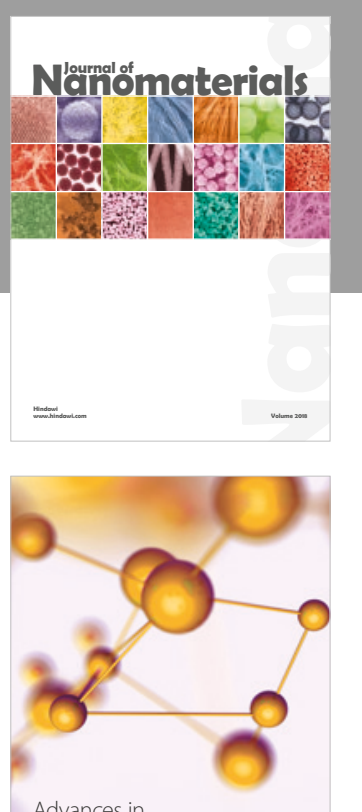

Physical Chemistry
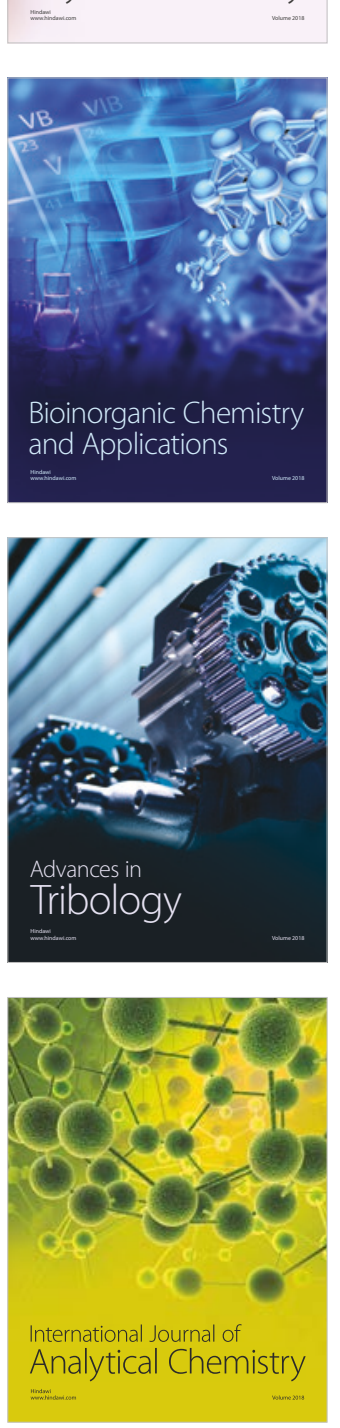

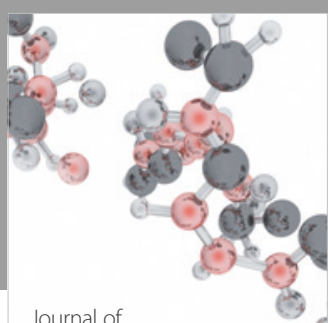

Analytical Methods

in Chemistry

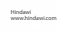

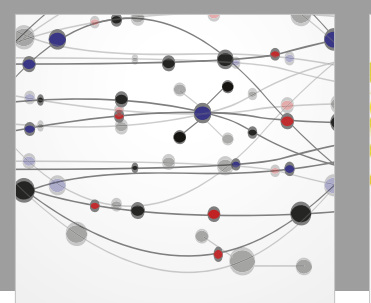

The Scientific World Journal

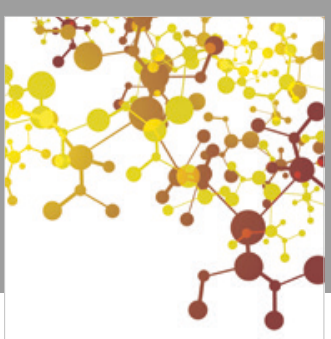

Journal of

Applied Chemistry
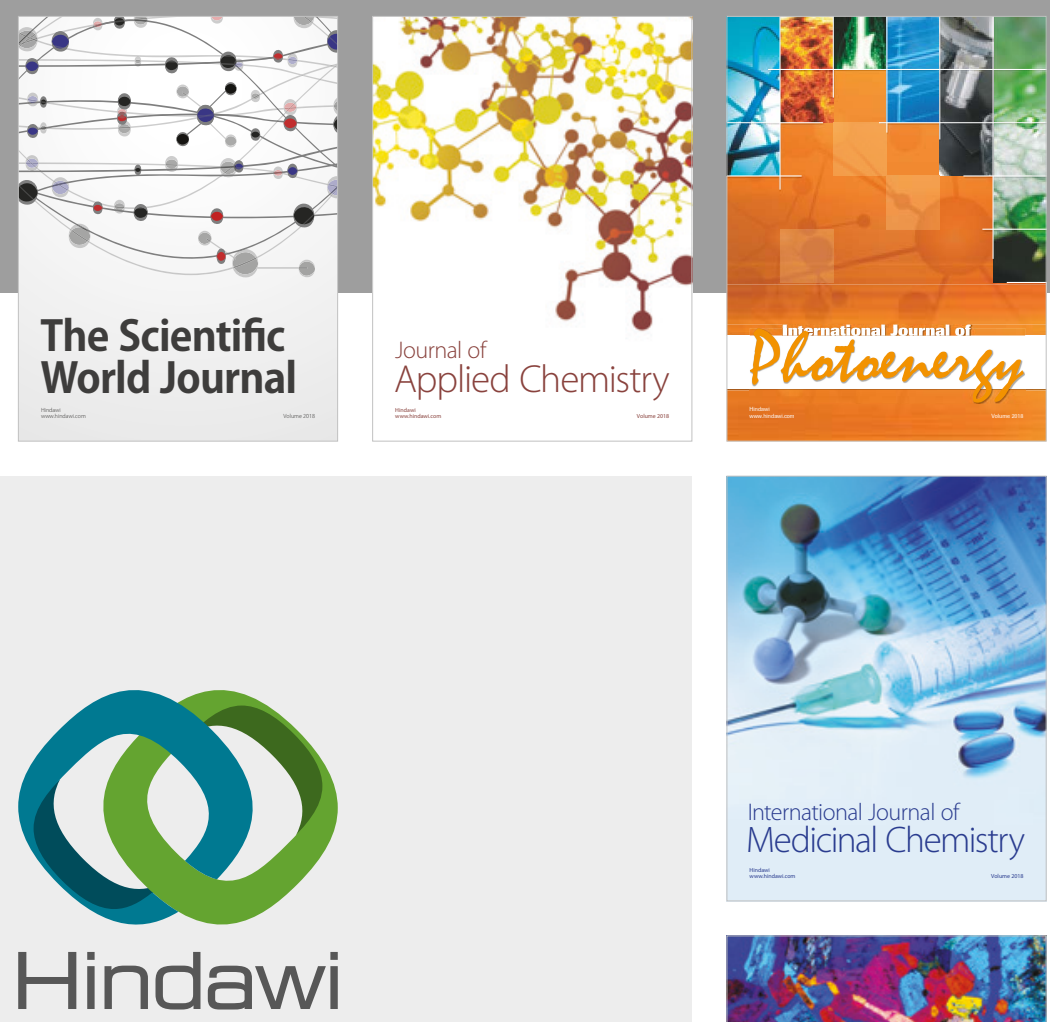

Submit your manuscripts at

www.hindawi.com
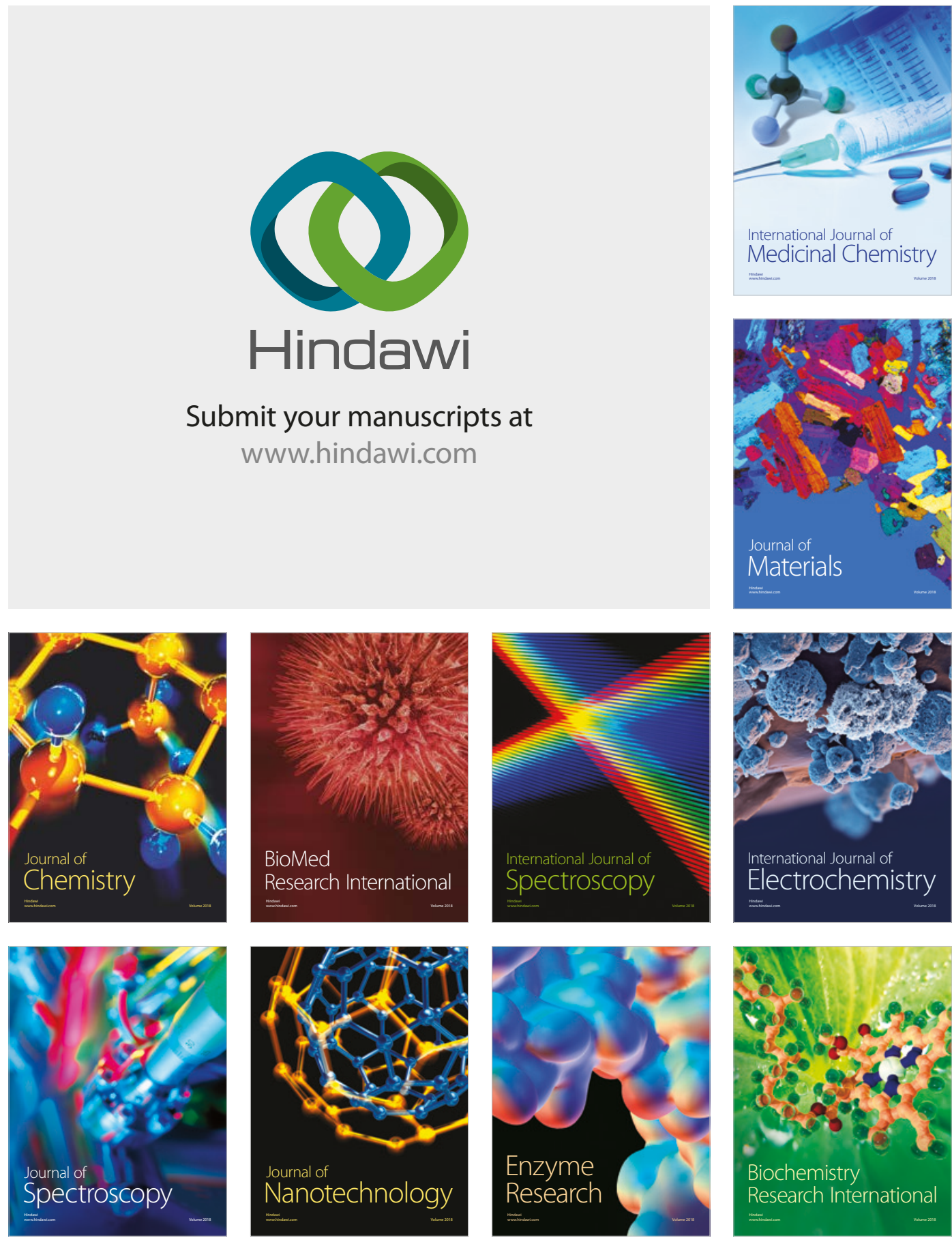
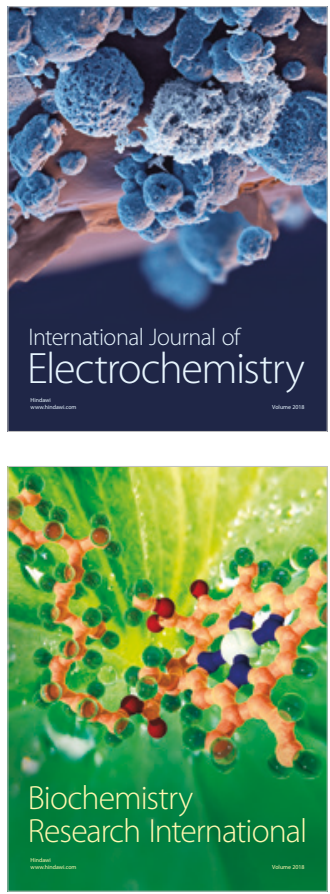